\title{
INHERITANCE OF CHROMOSOME NUMBER IN PIGS*
}

\author{
A. F. MCFEE AND M. W. BANNER \\ Agricultural Research Laboratory of the University of Tennessee $\dagger$, \\ Oak Ridge, Tennessee 37830
}

(Received 23rd Fanuary 1968, revised 25th May 1968)

\begin{abstract}
Summary. All possible crosses were made between European wild pigs with either 36 or 37 chromosomes and domestic swine with 38 . The $36 \times 36$ cross produced only pigs with 36 ; the $36 \times 37$ and $37 \times 38$ crosses yielded the parent numbers in about equal numbers of pigs. All pigs resulting from the $36 \times 38$ cross had 37 , while crossing $37 \times 37$ gave progeny with 36,37 or 38 in about a $1: 2: 1$ ratio. It is surmised that the three unpaired members in the 37-chromosome animal act as a trivalent during meiosis with two telocentric chromosomes behaving as a unit. No firm evidence indicated reduced fertility in any of the animals nor were any physical changes evident which could be associated with different chromosome forms.
\end{abstract}

\section{INTRODUCTION}

In a previous report (McFee, Banner \& Rary, 1966) we have described the existence among European wild pigs of variation in chromosome number between animals. The majority of these pigs possess 36 chromosomes rather than the 38 characteristic of domestic breeds while some, postulated to be crossbreds, have 37 . This report presents the results of a breeding programme testing the possible combinations of matings between animals with the three chromosome numbers.

\section{MATERIALS AND METHODS}

Chromosome numbers were determined from peripheral blood leucocytes cultured under phytohaemagglutinin stimulation (McFee, Banner \& Murphree, 1965). At least twelve well-spread metaphases from each animal were examined at a magnification of 1250 ; total chromosome number and the number of telocentric members were determined for each metaphase to arrive at modal values for the animal. Analyses were performed on all animals prior to their use for breeding. Females introduced into the programme were allowed to undergo at least one heat period before being mated in order to ensure unquestioned parentage. Sows were penned individually or in groups of two to four which

\footnotetext{
* Published with the permission of the Director of the University of Tennessee Agricultural Experiment Station, Knoxville.

† Operated by the Tennessee Agricultural Experimental Station for the U.S. Atomic Energy Commission under Contract No. AT-40-1-GEN-242.
} 
were being mated to the same boar. Individual pens were furnished for farrowing; the sex and colour pattern of each pig were recorded at birth. Most of the wild pigs used for breeding were unrelated first or second generation descendants of animals captured from the Tellico Wildlife Management Area herd. Of the sixty-two litters involved, however, nine resulted from half-sib matings between members of two crossbred litters in an effort to establish colour pattern trends. Matings were made over a 2-year period in a manner designed to yield approximately equal numbers of offspring from each of the five possible crosses involving 36- or 37-chromosome animals. A Pitman-Moore herd was used as a source of 38-chromosome individuals since their small size allows easier natural mating with wild pigs.

\section{RESULTS}

The litters produced from the various matings contained a total of 341 pigs. Our experience has indicated that leucocyte cultures from very young pigs are consistently of poor quality; therefore, the practice was adopted of sampling animals at about 3 to 4 weeks of age. Stillbirths and early postnatal losses thus

TABLE 1

GHROMOSOME NUMBER IN PIGS RESULTING FROM GROSSES BETWEEN PARENTS WITH VARIOUS NUMBERS

\begin{tabular}{l|rrr}
\hline & \multicolumn{4}{|c}{} \\
& \multicolumn{4}{|c}{ Pigs with 2 of parents } & equal to: \\
& 36 & 37 & 38 \\
\hline $36 \times 36$ & 45 & & \\
$36 \times 37$ & 19 & 27 & \\
$36 \times 38$ & & 56 & \\
$37 \times 37$ & 12 & 26 & 8 \\
$37 \times 38$ & & 21 & 35 \\
\hline
\end{tabular}

reduced the number of animals on which chromosome evaluations were made to 249. Modal chromosome numbers of pigs resulting from the various crosses are summarized in Table 1. Among a few hundred animals sampled we have never found a domestic pig with other than 38 chromosomes, so it can be assumed that the $38 \times 38$ cross produces only progeny with 38 chromosomes. Chromosome morphology within animals with the various numbers of chromosomes did not vary from that described previously. Thus, animals with 37 chromosomes had the same three unpaired members, one subtelocentric and two telocentrics, whether they were progeny of a $36 \times 37$ or any of the other three crosses capable of producing this number. Incidentally, kidney cells have been karyotyped from animals with each of the three complements and in all cases their chromosomal make-up was identical to that of leucocytes from the same animal.

The litters produced in the experiment contained an average of 5.5 pigs of which $23 \%$ were born dead; a summary of litters from the various crosses is contained in Table 2. It should be emphasized that values for the number of 
animals born dead represent all pigs dead at the time the litter was first seen and are not true reflections of stillbirth rates since they include several victims of maternal cannibalism and other hazards of immediate post-parturition life. Statistical analysis, by Duncan's Multiple Range procedure, showed that the $37 \times 38$ cross produced significantly larger litters $(P<0.05)$ than the other four matings but that the remaining four did not differ among themselves. No indication of imbalance of the sex ratio was seen since, of the total pigs analysed, 125 were males and 124 were females.

European wild pigs at birth normally show a distinct colour pattern of longitudinal, alternate light and dark body stripes changing to the agouti pattern as the animal matures; this is contrasted to the solid black characteristic of the Pitman-Moore. All the pigs born from $36 \times 36$ and $36 \times 37$ matings were distinctly

TABLE 2

LITTER SIZE AND YOUNG BORN DEAD

AMONG CROSSBRED PIGS WITH VARIOUS

CHROMOSOME NUMBERS

\begin{tabular}{c|c|c|c}
\hline $2 n$ of parents & $\begin{array}{c}\text { No. of } \\
\text { litters }\end{array}$ & $\begin{array}{c}\text { Pigs/ } \\
\text { litter }\end{array}$ & $\begin{array}{c}\% \text { Born } \\
\text { dead }\end{array}$ \\
\hline $36 \times 36$ & 12 & 5.4 & 23.1 \\
$36 \times 37$ & 15 & 4.6 & 24.6 \\
$36 \times 38$ & 11 & 5.5 & $8 \cdot 2$ \\
$37 \times 37$ & 13 & $5 \cdot 1$ & 37.9 \\
$37 \times 38$ & 9 & 7.9 & 16.9 \\
$(38 \times 38)^{*}$ & 20 & 6.5 & 15.4 \\
\hline
\end{tabular}

*Last twenty litters born in Pitman-Moore herd during period of this experiment included for comparison.

striped. Matings of the $36 \times 38$ and $37 \times 38$ type, i.e. having one Pitman-Moore parent, produced approximately twice as many black as striped pigs. Progeny exhibiting both colour patterns were produced by the $37 \times 37$ matings although striped pigs predominated. Disregarding the chromosome number of the parents, matings between striped individuals produced only striped pigs; when one parent was black, black pigs predominated by about two to one. When both parents were black but one or both carried some wild breeding, about one out of six progeny still had the typical striped pattern. Many of the black pigs from these crosses showed a very faint but quite regular striped pattern of slight variations in colour intensity. The crossbred pigs tended to retain the long nose, coarse hair and general body features of the wild pig in all the first generation crosses. If they were of the same colour pattern, the 38-chromosome pigs resulting from the $37 \times 37$ cross could not be distinguished from their littermates with 36 .

\section{DISGUSSION}

The occurrence of closely related animals with different chromosome numbers which will crossbreed and produce fertile offspring offers an unusual opportunity to draw conclusions relative to the behaviour of chromosomes during 
meiotic division. The karyotypic make-up of the European pig differs from that of domestic breeds in that it contains a pair of subtelocentric chromosomes, the long and short arms of which are morphologically similar to long and short telocentric pairs in domestic swine (Plate 1). Since all domestic breeds seem to have the same 38 chromosomes it may be concluded that early in their development a centric split of one pair occurred if, indeed, the European wild pig is their common ancestor (Zeuner, 1963). The 37-chromosome animal is of particular interest since it allows some deductions to be made relative to the behaviour of the three unpaired chromosomes. It would seem that during the meiotic division process the two telocentric members synapse with the two arms of the subtelocentrics as though collectively they represented a single chromosome homologous to the unpaired subtelocentric. If they behaved independently then one would expect that, at the reductional division, the chromosomes could be unevenly divided. This would, in turn, lead to fertilization events involving extra chromosomes or a shortage. Animals with extra chromosomes were not seen among the progeny of any of the crossbreds. The loss of a chromosome is considered to be a lethal event to the cell and should cause decreased fertility or, depending on the frequency of occurrence, sterility of the animal. At first observation the average litter sizes would seem quite low and indicative of lethal factors. It must be remembered, however, that these are litters out of Pitman-Moore and wild sows in which the average litter sizes are six (Bustad, Horstman \& England, 1966) and four (Lush, 1921), respectively. The ten litters sired by wild boars and farrowed by Pitman-Moore sows contained an average of 9.0 pigs; conversely, the same number of litters out of wild sows and sired by Pitman-Moore boars averaged only $4 \cdot 2$ pigs each. This litter size relationship does not offer any firm indication of lethality due to chromosome loss but suggests that the small litters obtained were due to the predominance of wild breeding in the experiment. Neither do the high percentages of pigs tabulated as born dead in three of the groups indicate death as a result of chromosome shortage since this condition would probably be expressed shortly after fertilization. Their magnitude is due largely to the fact that wild sows in captivity are poor mothers, prone to be quite excitable and often cannibalistic. Since most of the deaths occurred before the age at which samples were routinely taken, little information is available regarding the susceptibility of pigs with various chromosome numbers to early postnatal death. The few animals tested before an early death were, however, distributed in all three karyotypic classes.

It becomes readily apparent that the chromosome number in progeny of matings between parents with 36,37 or 38 can be predicted on a Mendelian ratio basis. The 36- and 38-chromosome animals can produce only one type of gamete each compared to the two types possible in pigs with 37 . Therefore a mating between parents with 36 and 38 chromosomes can result only in pigs which have 37 . The $36 \times 37$ and $37 \times 38$ matings should yield pigs having their parents' numbers in a $1: 1$ ratio while the $37 \times 37$ mating should produce pigs with 36,37 or 38 in a $1: 2: 1$ ratio. When each type mating was tested separately, the results agreed within statistically acceptable limits with these predictions (in all cases $\chi^{2}>0 \cdot 10$ ). 


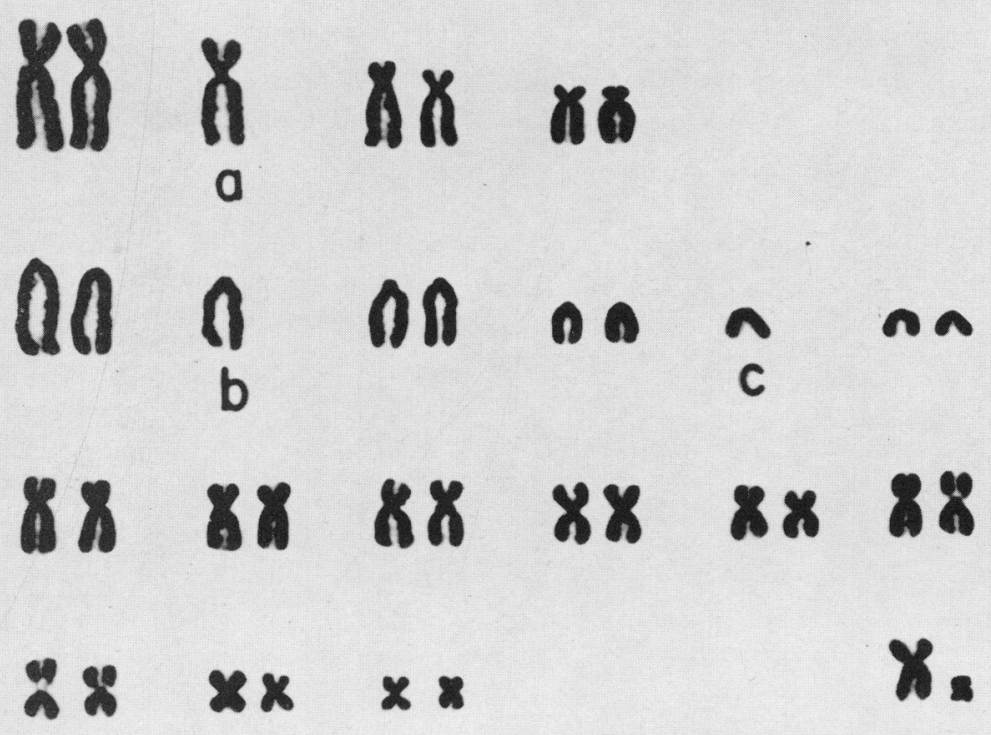

Karyotype of a 37-chromosome hybrid pig; note three unpaired members. In the 'purebred' wild pig a chromosome homologous to the subtelocentric (a) exists and the unpaired telocentrics (b and c) are absent, resulting in a 36-chromosome karyotype. Domestic pigs have 38 chromosomes as a result of the absence of the subtelocentric and the presence of homologues to the two telocentrics.

(Facing p. 12) 
The colour patterns exhibited by pigs in this study serve to emphasize the complexity of colour inheritance in swine which was recognized long ago by Wright (1918) and by Wentworth \& Lush (1923). We would take exception to the statement of Wentworth \& Lush that the striped pattern of wild pigs is dominant over all colours except white and the white belt. Certainly it is not clearly dominant over the Pitman-Moore black and in a single litter from a Yorkshire sow bred to a wild boar (not part of this experiment) we observed three white pigs and three distinctly marked with wild pig stripes. The faint striping seen in some black pigs would, however, support their contention that the wild pig carries a factor for the striped pattern and a separate factor which intensifies that pattern. Chromosomal polymorphism such as occurs in these pigs suggests the possibility of gene changes as a result of centric fission. Although such events are certainly not ruled out, we are unable to identify any characteristic whose mode of inheritance would indicate such an occurrence in these animals.

While chromosome polymorphism has occasionally been reported in domestic animals, its effect on fertility and the production of hybrid animals has not been thoroughly elucidated. The retention of the basic number of chromosome arms (fundamental number or NF of Matthey, 1965) at least among the autosomes, would seem to be essential to the production of fertile hybrid offspring. Among the three types of pigs in this study the NF is constant at sixty-four. An apparent condition of centric fusion has recently been described by Gustavsson (1966) in a breed of Swedish cattle. Out of 1134 animals tested, four apparently fertile bulls exhibited a pair of subtelocentric chromosomes which seemed to have resulted from the fusion of two telocentric pairs. The expected condition of three unpaired chromosomes if these bulls were mated to normal cows was indeed found in 122 animals; however, all showed NF equal to sixty-two. Kieffer \& Cartwright (1968) have examined the chromosomes of Brahman cattle and found that they constitute a specialized case of inconsistent NF. In Brahman bulls the $Y$ chromosome is telocentric rather than having the metacentric form of European breeds; NF is thus reduced from sixty-two to sixty-one. Whether the fertility of these males and their hybrid offspring is due to the retention of all the $\mathrm{Y}$ chromosome material by pericentric inversion or the loss of non-essential material is not yet known.

There is certainly more than the constancy of chromosome arm number involved in the production and fertility of interspecific hybrids. Benirschke, Malouf \& Low (1965) described karyotypic differences between the domestic horse, Equus caballus, and its supposed ancestor, Equus przewalskii Poliakoff, which would make their hybridization seem unlikely to occur. Koulischer \& Frechkop (1966) have, however, shown that hybridization between these two species not only occurs but can produce fertile offspring. The latter authors present a karyotype for the domestic horse which is considerably less divergent from przewalskii and therefore more compatible with interspecies crossing. With a difference of only one telocentric pair of autosomes between the parent lines the hybrid has the expected unpaired telocentric. Since the karyotype of the $F_{2}$ was not presented, it is impossible to draw conclusions about the meiotic behaviour of an unpaired chromosome where no homologous arm exists. It 
would be extremely interesting to see the results of this hybrid backcrossed on the other (przewalskii) parent line and to have more information on the overall fertility of the hybrids.

There is evidently a limit to the divergence between chromosome complements of parent lines which will allow crossbreeding and retain fertility of the progeny. In the mule, Benirschke, Brownhill \& Beath (1962) show nine and Trujillo, Stenius, Christian \& Ohno (1962) indicate at least seven chromosomes which have no homologue. Thus, a hybrid can be produced by crossing the horse and donkey even though their total chromosome arm numbers differ by some ten or twelve. The disparity between maternal and paternal sets of chromosomes, however, seems to interfere with meiotic divisions and causes the hybrid to be sterile. The sheep and goat, on the other hand, have the same chromosome arm number which, in turn, is characteristic of their hybrid embryos (Buttle \& Hancock, 1966; Hancock \& Jacobs, 1966), still these embryos seem invariably to die during the early embryonic period. While the present report emphasizes the fact that some divergence of chromosome arrangement is compatible with the production of fertile progeny, the question still remains of just how much is permissible.

\section{ACKNOWLEDGMENT}

Our sincere thanks go to $\mathrm{Mr}$ Bill Kyle for his help in managing the animals used in this study.

\section{REFERENCES}

Benirschke, K., Brownhill, L. E. \& Beath, M. M. (1962) Somatic chromosomes of the horse, the donkey and their hybrids, the mule and the hinny. F. Reprod. Fert. 4, 319.

Bentrschke, K., Malouf, N. \& Low, R. J. (1965) Chromosome complement: Differences between Equus caballus and Equus przewalskii Poliakoff. Science, N.Y. 148, 382.

Bustad, L. K., Horstman, V. G. \& England, D. C. (1966) Development of Hanford miniature swine. In: Swine in Biomedical Research. Eds. L. K. Bustad and R. O. McClellan. Frayn Printing Co., Seattle, Washington.

Buttle, H. L. \& Hancock, J. L. (1966) The chromosomes of goats, sheep and their hybrids. Res. vet. Sci. 7, 230.

Gustavsson, I. (1966) Chromosome abnormality in cattle. Nature, Lond. 211, 865.

Hancock, J. L. \& JaCoss, P. A. (1966) The chromosomes of goat $\times$ sheep hybrids. F. Reprod. Fert. 12,591 .

KiffFer, N. M. \& Cartwrighr, T. C. (1968) Sex chromosome polymorphism in domestic cattle. $\mathcal{F}$. Hered. 59, 35.

Koulischer, L. \& Frechrop, S. (1966) Chromosome complement: A fertile hybrid between Equus prjewalskii and Equus caballus. Science, N.Y. 151, 93.

Lush, J. L. (1921) Inheritance in swine. 7. Hered. 12, 57.

MatTHEy, R. (1965) Cytogenic mechanisms and speciation of mammals. In: The Chromosomes: Structural and Functional Aspects. Ed. G. Yerganian. Symposium of the Tissue Culture Association.

McFee, A. F., Banner, M. W. \& Murphree, R. L. (1965) Chromosome analysis of peripheral leucocytes of the sheep. 7. Anim. Sci. 24, 551 .

MaFeE, A. F., BANNER, M. W. \& RARY, J. M. (1966) Variation in chromosome number among European wild pigs. Cytogenetics, 5, 75.

Trujillo, J. M., Stenius, C., Christian, L. G. \& Ohno, S. (1962) Chromosomes of the horse, the donkey, and the mule. Chromosoma, 13, 243.

WeNTwORTh, E. N. \& Lush, J. L. (1923) Inheritance in swine. F. agric. Res. 23, 557.

WRIGHT, S. (1918) Color inheritance in mammals. VIII. Swine. F. Hered. 9, 33.

ZEUNER, F. E. (1963) A history of domesticated animals, p. 268. Harper \& Row, New York. 